\title{
Identification of Plant Viruses Infecting Pear Using RNA Sequencing
}

\author{
Nam-Yeon Kim ${ }^{1}$, Hyo-Jeong Lee ${ }^{1}$, Hong-Sup Kim², Su-Heon Lee ${ }^{3}$, Jae-Sun Moon ${ }^{4}$, and Rae-Dong Jeong (iD ${ }^{\text {* }}$ \\ ${ }^{I}$ Department of Applied Biology, Institute of Environmentally Friendly Agriculture, Chonnam National University, \\ Gwangju 61185, Korea \\ ${ }^{2}$ Seed Testing \& Research Center, Korea Seed \& Variety Service, Gimcheon 39660, Korea \\ ${ }^{3}$ School of Applied Biosciences, Kyungpook National University, Daegu 98411, Korea \\ ${ }^{4}$ Plant Genome Research Center, Korea Research Institute of Biosciences \& Biotechnology, Daejeon 34141, Korea
}

(Received on January 25, 2021; Revised on March 30, 2021; Accepted on April 23, 2021)

Asian pear (Pyrus pyrifolia) is a widely cultivated and commercially important fruit crop, which is occasionally subject to severe economic losses due to latent viral infections. Thus, the aim of the present study was to examine and provide a comprehensive overview of virus populations infecting a major pear cultivar ('Singo') in Korea. From June 2017 to October 2019, leaf samples $(n=110)$ of pear trees from 35 orchards in five major pear-producing regions were collected and subjected to RNA sequencing. Most virus-associated contigs matched the sequences of known viruses, including apple stem grooving virus (ASGV) and apple stem pitting virus (ASPV). However, some contigs matched the sequences of apple green crinkle-associated virus and cucumber mosaic virus. In addition, three complete or nearly complete genomes were constructed based on transcriptome data and subjected to phylogenetic analyses. Based on the number of virus-associated reads, ASGV and ASPV were identified as the dominant viruses of 'Singo.' The present study describes the virome of a major pear cultivar in Korea, and looks into the di-

*Corresponding author.

Phone) +82-62-530- 2075, FAX) +81-62-530-2069

E-mail)jraed2@jnu.ac.kr

ORCID

Rae-Dong Jeong

https://orcid.org/0000-0002-6370-8909

Handling Editor : Ju-Yeon Yoon

(c) This is an Open Access article distributed under the terms of the Creative Commons Attribution Non-Commercial License (http:// creativecommons.org/licenses/by-nc/4.0) which permits unrestricted noncommercial use, distribution, and reproduction in any medium, provided the original work is properly cited.

Articles can be freely viewed online at www.ppjonline.org. versity of viral communities in this cultivar. This study can provide valuable information on the complexity of genetic variability of viruses infecting pear trees.

Keywords : pear, plant viruses, RNA sequencing, viral population

Under natural conditions, plants are exposed to attacks by various pathogens, including fungi, bacteria, nematodes, viruses, and viroids. In particular, viruses are considered economically important because of their abundance in numerous crops (Akinyemi et al., 2016). Therefore, early virus identification is paramount to reduce crop losses and prevent viral disease spread.

Among the many plant species affected by viruses, Asian pear (Pyrus pyrifolia) is a widely cultivated and commercially important fruit crop in several regions worldwide, including Argentina, China, Italy, Korea, and Japan (Saito, 2016). Pear is one of the major pome fruit crops, with 10,000 ha of area under cultivation (Yoon et al., 2014). Over 3,000 pear cultivars are grown worldwide; however, a few common and economically important cultivars with different harvest times are grown in Korea (Elzebroek and Wind, 2008).

Pears are generally propagated via cloning and grafting to maintain cultivar traits; therefore, viruses and viroids that infect pear trees are readily transmitted. Major viruses infecting pear trees include apple chlorotic leaf spot virus (Trichovirus), apple stem grooving virus (ASGV; Capillovirus), and apple stem pitting virus (ASPV; Foveavirus) (Mahfoudhi et al., 2013). Although most viral infections of pear are asymptomatic, ASGV frequently causes black necrotic leaf spot disease symptoms, leading to significant 
economic losses in 'Niitaka' pear (Cho et al., 2010). In addition, multiple viruses and viroids simultaneously infect pear trees, potentially further reducing overall production.

At present, the detection of viruses and viroids typically involves the observation of viral disease symptoms, followed by the application of direct detection methods, such as enzyme-linked immunosorbent assay (ELISA), realtime polymerase chain reaction (RT-PCR), loop-mediated isothermal amplification, and recombinase polymerase amplification (Cho et al., 2010; Kim et al., 2018; Lu et al., 2018). However, these methods rely on prior information on the potential viruses and are only suitable for detecting known viruses and viroids and not for identifying novel viral pathogens.

Advances in next-generation sequencing (NGS)-based approaches have furthered our understanding of viral genome variability and evolution as well as facilitated the identification of viral disease mechanisms, measurement of viral abundance and diversity, screening of asymptomatic viruses, detection of low viral titers in infected plants, and discovery of unknown viruses, thereby providing novel insights into the complexity of the viral biosphere. Several studies have previously used NGS to investigate viromesdefined as the collective genomes of viruses present in a specific host or environment - and documented the viromes of several crops (Akinyemi et al., 2016; Jo et al., 2017; Jo et al., 2018a, 2020). Therefore, high-throughput sequencing (HTS) can be integrated into the diagnostics and management of viral diseases, even when previously unknown viruses are involved. However, despite the increasing adverse effects of viruses on pear production, relatively little is known about viruses and viroids that infect this fruit crop. To this end, the aim of the present study was to employ metatranscriptomics for investigating RNA viruses infecting the most commercially important pear cultivar 'Singo' (SG) in Korea.

\section{Materials and Methods}

Plant materials. Pear leaves were collected from 35 orchards in five major pear-producing regions (Naju, Chonan, Namyangju, Ulsan, and Sangju) in Korea during the summer and fall seasons of 2017-2019 (Fig. 1A). The collection of these pear leaves was permitted by the owners of the orchards. Pear leaves with typical disease symptoms, such as deformation, chlorotic or necrotic leaf spots, and yellow veins, were collected from 100 SG trees (Fig. 1B). The collected SG leaf samples were pooled, ground in liquid nitrogen, and stored at $-80^{\circ} \mathrm{C}$.
RNA extraction and library preparation for RNA sequencing. Total RNA was extracted from the ground samples using the IQeasy Plus Plant RNA Extraction Mini Kit (iNtRON, Daejeon, Korea) according to the manufacturer's instructions, and the quality and quantity of the extracted RNA samples were measured using Agilent 2100 Bioanalyzer (Agilent, Santa Clara, CA, USA) and gel electrophoresis. Libraries were prepared from rRNA-depleted RNA samples using the TruSeq Stranded Total RNA Library Prep Kit (Illumina, San Diego, CA, USA) according to the manufacturer's instructions. Briefly, following rRNA removal, the remaining RNA was chemically fragmented and random primed for reverse transcription. The average insert size of the library was $200 \mathrm{bp}$. Then, adapters were ligated, and PCR amplification was performed to selectively enrich the DNA fragments with adapters and amplify the library DNA. Agilent 2100 Bioanalyzer was used for quality control of the generated library, and the prepared library was sequenced by Macrogen Co. (Seoul, Korea) using HiSeq 4000 sequencer, generating 200-bp paired-end reads.

Transcriptome assembly and analysis. Before analyzing the transcriptomes of SG, the infection of several pear leaf samples by ASGV and ASPV was investigated by RTPCR, which indicated that most pear trees were infected by either of the two viruses (data not shown). Transcriptome was assembled de novo using a workstation (two six-core CPUs and 256-GB RAM) with the Ubuntu 12.04.5 LTS operation system. Raw data obtained from the library were subjected to de novo transcriptome assembly. In addition, FASTQ files of the paired-end sequences from the library were subjected to de novo transcriptome assembly using gsAssembler v2.8 (Roche Diagnostics, Branford, CT, USA) with default parameters (Wylie et al., 2014). To identify viral sequences in the library, the assembled transcriptome contigs were compared with sequences in the viral reference database in NCBI (downloaded from https://www.ncbi.nlm.nih.gov/genome/viruses/) using MEGABLAST, which is markedly faster and more reliable for virus identification than other sequence similarity programs; the E-value cut-off was $1 \mathrm{e}^{-5}$. Following initial analysis, putative virus-associated contigs with mean sequence coverage values of $<10$-fold were discarded, and the remaining putative virus-associated contigs were compared with sequences in the NCBI NR (non-redundant proteins) database using tBLASTx. Finally, endogenous viruslike sequences were removed from the dataset, and virusassociated contigs were retained for further analyses. 


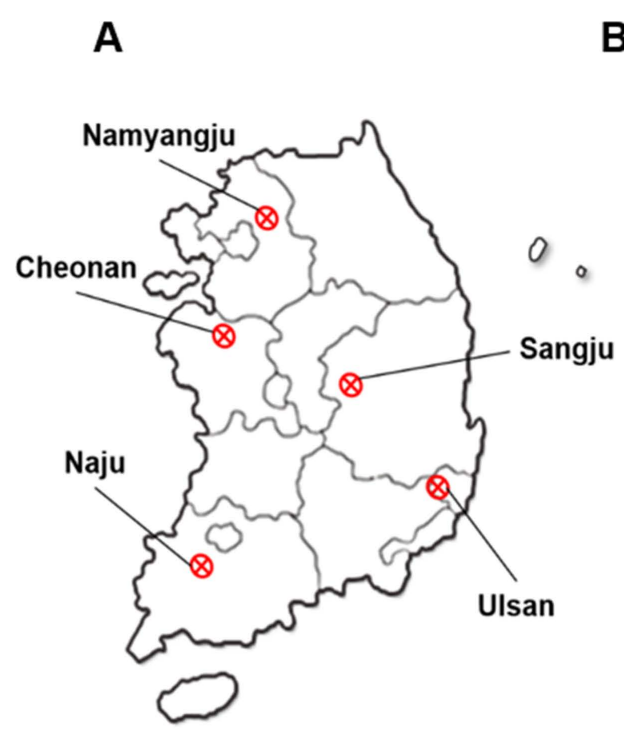

B

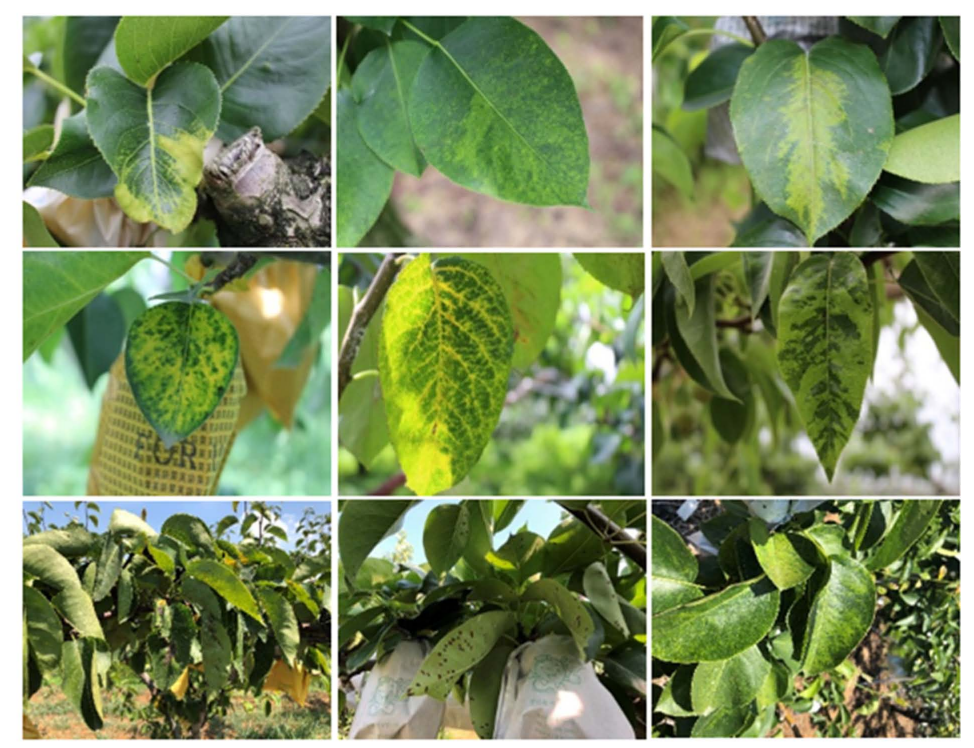

\section{C}

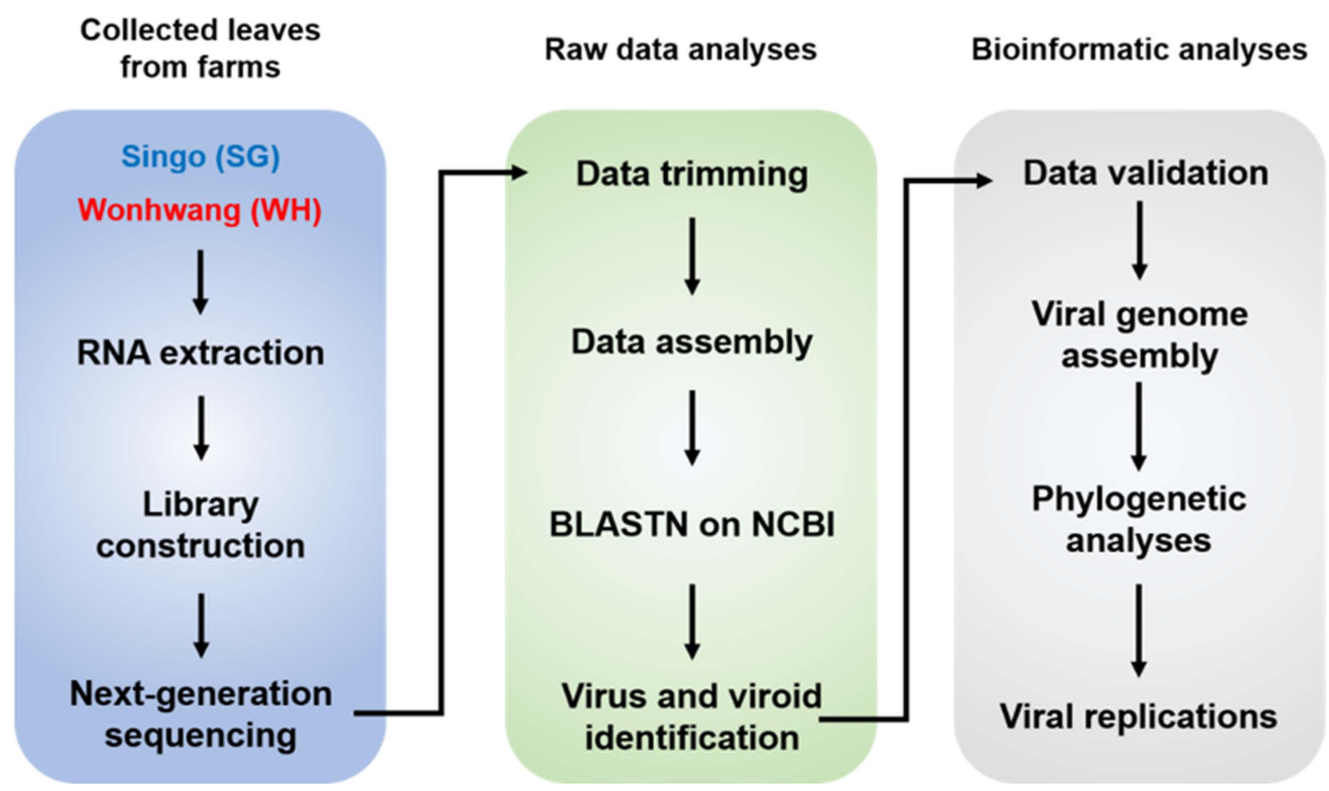

Fig. 1. Experimental procedure for identification of viruses infecting pear using RNA sequencing. (A) Sampling sites in Korea. (B) Symptoms of viral infection in pear trees in Korea. (C) Experimental scheme for investigating pear virome.

Viral sequence mapping and genome assembly. To generate complete viral genomes, the pear transcriptome sequences were compared with a complete reference viral genome sequence using BLAST. Contigs with the greatest similarity to specific viruses were selected. The virusassociated contigs were mapped to the reference viral genome using Geneious v. 11.1.5 (Kearse et al., 2012), and consensus sequences for each mapping file were generated using a threshold of $95 \%$ identity.
Phylogenetic analysis. The complete genome sequences obtained through the preceding analyses were used to construct phylogenetic trees. The complete coat protein (CP) gene sequences of $20 \mathrm{ASGV}$ isolates and $19 \mathrm{ASPV}$ isolates were used for phylogenetic analyses. NCBI accession numbers of all viral genomes are indicated in the phylogenetic trees. Additional complete viral genome sequences were obtained for analysis by comparing the sequenced viral genomes with the ones in GenBank using BLASTN and by removing partial sequences from the results. Nucleotide se- 
quences were aligned using CLUSTALW in BioEdit, and phylogenetic trees were generated using 1,000 bootstrap replicates based on either neighbor-joining or Kimura 2-parameter method in MEGA 5.05 (Tamura et al., 2011). Pairwise distances were calculated using the PASC algorithm, available from GenBank (Bao et al., 2012).

Calculation of viral abundance and copy number. To calculate viral abundance, MEGABLAST was performed against the sequenced reads associated with respective viruses. FASTQ files were converted to FASTA files using the FASTX-Toolkit (http://hannonlab.cshl.edu/fasx toolkit/), and the resulting FASTA files were used for performing MEGABLAST. Copy number was calculated by multiplying the number of viral reads by $150 \mathrm{bp}$ (average length of total reads) and then dividing the product by the total length (bp) of individual genomes (Jo et al., 2017).

Confirmation of the presence of identified viruses by RT-PCR. To confirm the presence of identified viruses in pear samples, RT-PCR was performed using virus-specific primer sets (Table 1). One-tube multiplex RT-PCR was performed in $20 \mu \mathrm{l}$ reactions containing SuprimeScript RTPCR Premixture (GeNet Bio, Daejeon, Korea), $2 \mu \mathrm{l}$ RNA template, forward and reverse primers, and DEPC water. RT-PCR was conducted under the following conditions: $50^{\circ} \mathrm{C}$ for $30 \mathrm{~min} ; 95^{\circ} \mathrm{C}$ for $5 \mathrm{~min} ; 38$ cycles of $95^{\circ} \mathrm{C}$ for $30 \mathrm{~s}$, $58^{\circ} \mathrm{C}$ for $30 \mathrm{~s}$, and $72^{\circ} \mathrm{C}$ for $60 \mathrm{~s}$; and $72^{\circ} \mathrm{C}$ for $5 \mathrm{~min}$. The resulting amplification products were subjected to electrophoresis on $2 \%$ agarose gel in $1 \times$ TAE buffer, stained using ethidium bromide, and visualized under UV light.
Product size was determined by comparison with a $100 \mathrm{bp}$ DNA ladder (iNtRON).

Data availability. The raw dataset generated in the present study will be available, upon publication, in the NCBI Sequence Read Archive (SRA) repository under accession number SRR8466619. The viral genome sequences generated in the present study are deposited in GenBank with respective accession numbers.

\section{Results}

Library construction. Transcriptome datasets were generated from the library using paired-end sequencing. For viral metatranscriptomic analysis, transcriptome data were analyzed according to the experimental scheme (Fig. 1C). Raw RNA sequencing (RNA-Seq) data corresponding to the library are deposited in the SRA repository (Supplementary Table 1). A total of $6.7 \mathrm{Mb}$ data were obtained from the SG library, corresponding to $78,624,272$ reads.

Virus identification. Raw reads from the library were de novo assembled using gsAssembler. The N50 values ranged from 993 to 1,126 bp (Supplementary Table 1). Moreover, 111,724 small ( $\geq 200 \mathrm{bp}$ ) and 35,725 large ( $\geq 500 \mathrm{bp}$ ) contigs were generated for the SG library (Fig. 2A).

The assembled contigs were compared with the sequences in the virus and viroid reference databases using BLAST. A total of 1,428 virus- and viroid-associated contigs were identified. After removing endogenous virus-
A

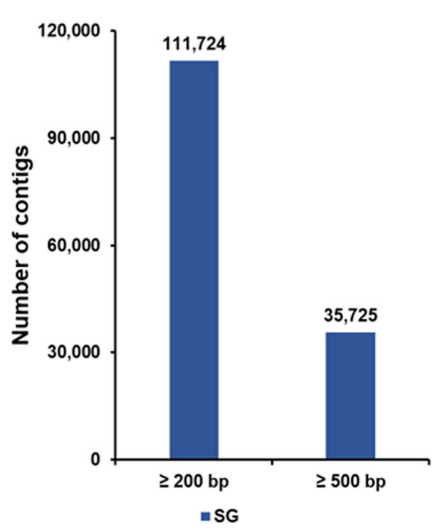

B

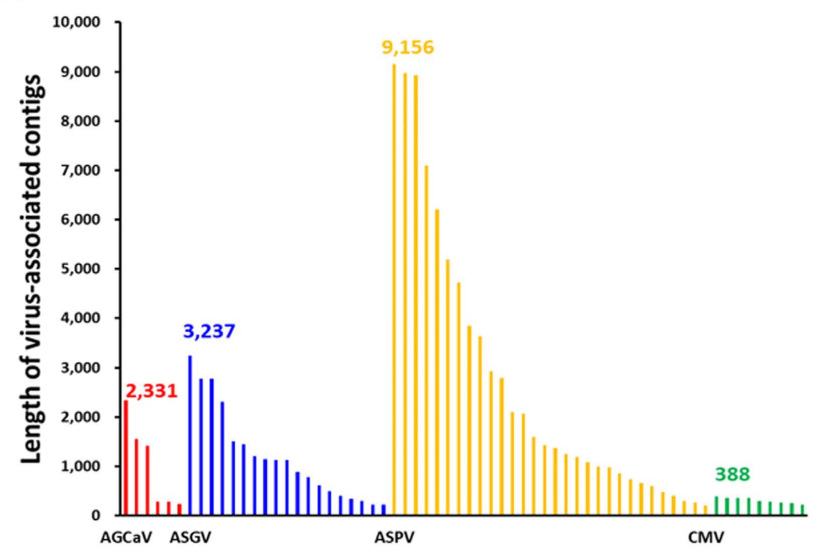

C

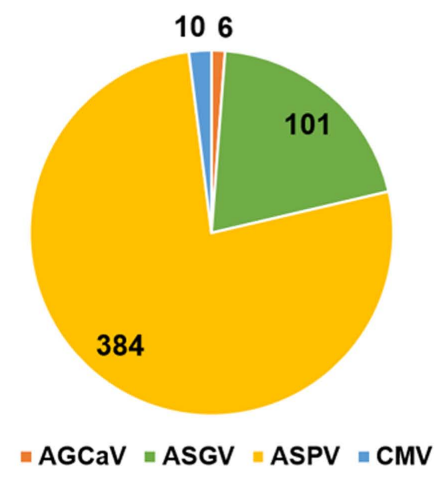

Fig. 2. Identification of viruses infecting pear trees using next-generation sequencing. (A) Contigs were de novo assembled from libraries of the pear cultivar 'Singo' (SG). (B) Size of contigs associated with the identified virus from SG library. Number indicates the length of the longest contig from the transcriptome. (C) The portion of the identified viruses according to the number of the assembled virusassociated contigs from the SG library. AGCaV, apple green crinkle associated virus; ASGV, apple stem grooving virus; ASPV, apple stem pitting virus; CMV, cucumber mosaic virus. 
A

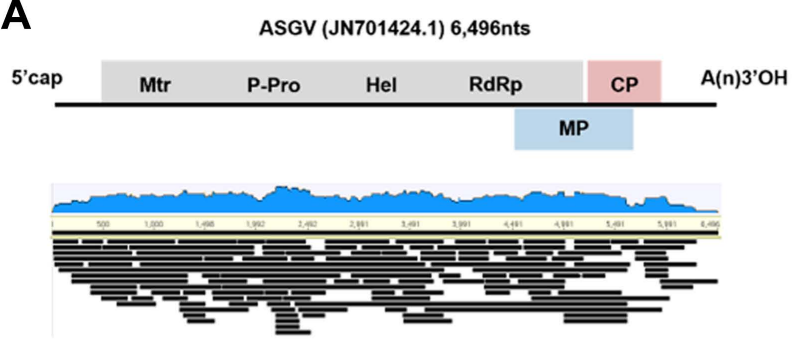

B

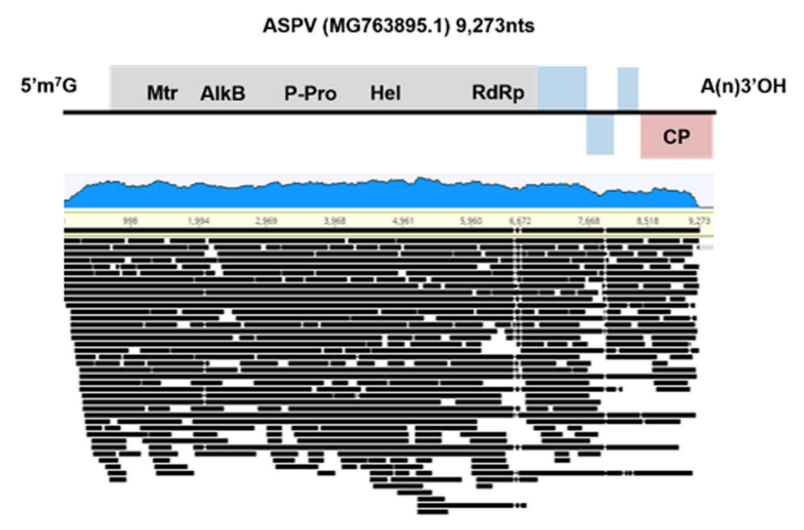

C

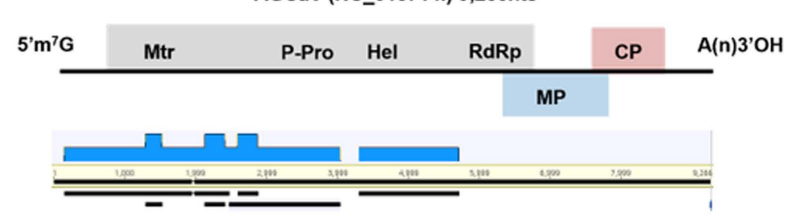

D
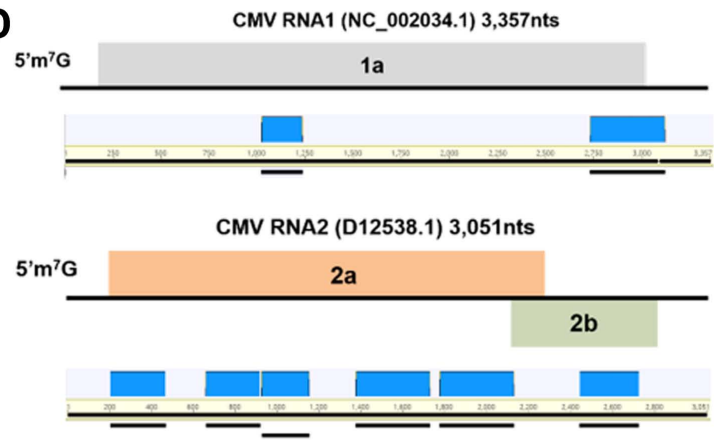

CMV RNA3 (AM183119.1) 2,192nts

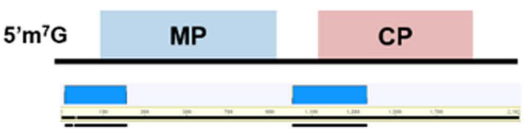

Fig. 3. The de novo genome assembly of identified viruses using pear transcriptome data. The genome organization of assembled viruses with accession number and the size of corresponding reference viral genomes and the alignment of associated contigs derived from pear transcriptome on each virus reference genome for ASGV (A), ASPV (B), AGCaV (C), and CMV (D). AGCaV, apple green crinkle associated virus; ASGV, apple stem grooving virus; ASPV, apple stem pitting virus; CMV, cucumber mosaic virus; $\mathrm{CP}$, coat protein.

associated sequences, 521 virus-associated contigs were identified.

The virus-associated contigs from the dataset were first combined and then classified. The viruses identified from the transcriptomes included members of two virus families, namely Betaflexiviridae (511 contigs, 98.1\%) and Bromoviridae (10 contigs, $1.9 \%$ ). The length of virusassociated contigs ranged from 200 to 9,156 bp (Fig. 2B), and the largest seven contigs ( $>5,000$ bp in length) likely represented the de novo assembled partial or nearly complete viral genomes. The virus-associated contigs from the SG library matched four reference viruses (Supplementary Table 2). In the SG library, ASPV-associated contigs ( $n$ $=384)$ were the most abundant, followed by ASGV- $(\mathrm{n}=$ $101)$, cucumber mosaic virus (CMV)- $(n=10)$, and apple green crinkle associated virus (AGCaV)-associated contigs $(n=6)$ (Fig. 2C).

De novo assembly of viral genomes. To assemble complete or nearly complete viral genomes from the transcrip- tome data, the virus-associated contigs were mapped to the corresponding reference genomes (Fig. 3). Some ASGVand ASPV-associated contigs covered the nearly complete genomes of the two viruses (Fig. 3A and B). Sizes of the virus-associated contigs ranged from 201 to 3,237 nt for ASGV and from 200 to 9,156 nt for ASPV (Supplementary Table 2). However, some sequence regions in genomes of the identified viruses were partially mapped to the virusassociated contigs (Fig. 3C and D). The size of AGCaVassociated contigs ranged from 236 to 2,331 nt and that of CMV-associated contigs ranged from 212 to $388 \mathrm{nt}$ (Supplementary Table 2). In case of $\mathrm{AGCaV}$, partial sequences were obtained from SG.

Phylogenetic relationships among identified viruses. To determine the phylogenetic relationships among the identified viruses and their congeners, phylogenetic trees were constructed for each virus using the obtained nearly complete genomes based on the respective homologous sequence or viral genome. The assembled viral genomes 
A

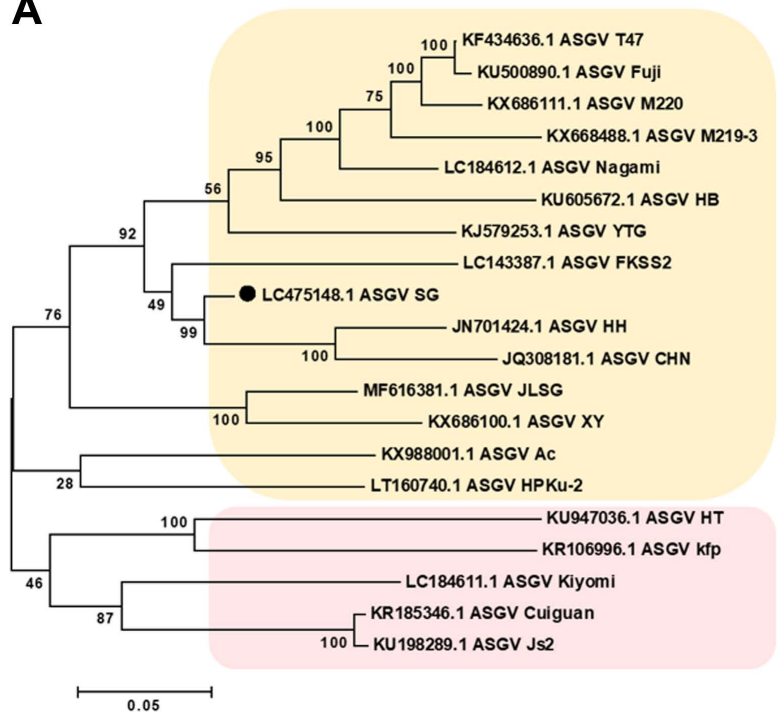

B

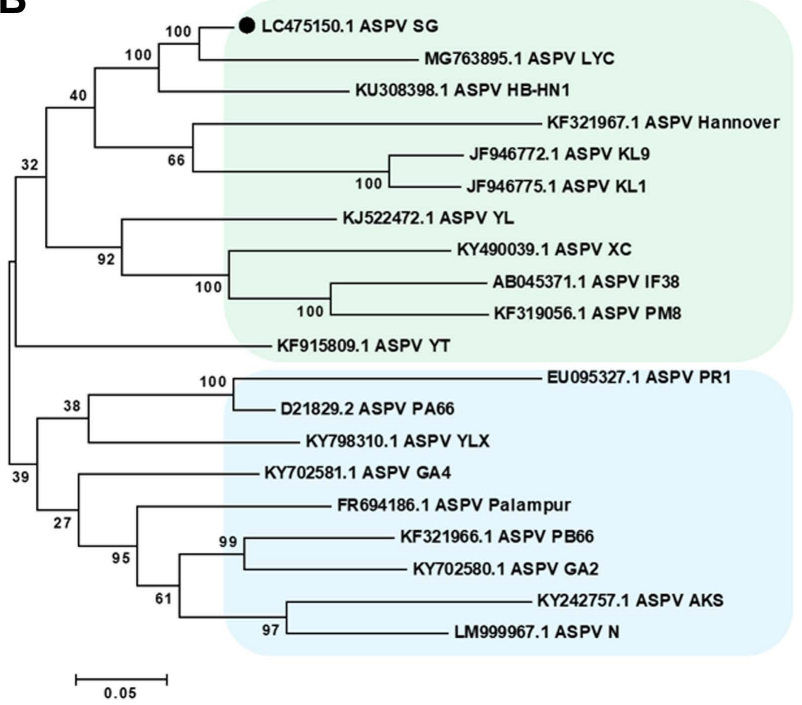

Fig. 4. Phylogenetic relationships of two viruses detected in pear transcriptome. Phylogenetic trees for ASGV (A), and ASPV (B) were constructed using MEGA 5.05. Phylogenetic analysis included consensus genome sequences generated using sequences from the 'Singo' library and reference genome sequences from GenBank based on the neighbor-joining methods and Kimura 2-parameter model. Bootstrap values along the branches are supported are by 1,000 replicates. The scale bar indicated the number of substitutions per residue. ASGV, apple stem grooving virus; ASPV, apple stem pitting virus.

were compared with sequences in the NT database using BLASTN. Owing to their high similarity between AGCaV and ASPV and incomplete assembly of AGCaV, phylogenetic analysis was performed with the complete sequences of ASGV isolates and ASPV isolates. The resulting phylogenetic tree indicated that the complete sequences included in the ASGV tree were separated into three clades. In the first clade, the ASGV isolate (accession no. LC475148) from the SG library was clustered with ASGV isolates derived from apple in China (Fuji, HB, T47, M220, and $\mathrm{HH}$ ) (Fig. 4A). The second clade included ASGV isolates from citrus and pear in China (Cuiguan, JLSG, kiyomi, and Js2). The third clade included ASGV isolates from apple and pear in China (YTG, Ac, HT, and kfp). Similarly, complete sequences included in the ASPV tree, generated using a ASPV genome obtained in the present study and 18 additional genomes from GenBank (Fig. 4B), were separated into two clades. Interestingly, the ASPV isolate (accession no. LC475150) from the SG library was clustered with ASPV isolates from apple and pear in China.

Viral diversity. To calculate viral abundance, raw transcriptome sequence data were directly compared with reference genome sequences for the identified viruses using BLAST. The SG transcriptome (178,174 reads) contained numerous virus-associated reads (Fig. 5A). Furthermore, ASPV (124,492 reads) and ASGV (52,945 reads) were the dominant viruses in the SG library (Fig. 5B).

To determine the amount of individual viral RNA in the transcriptome based on read number, the number of total reads was divided by the number of virus-associated reads and multiplied by $151 \mathrm{bp}$. ASPV (69.9\%) and ASGV (29.7\%) were the dominant viruses in the SG library, followed by AGCaV (0.4\%) and CMV (0.1\%) (Fig. 5C).

Furthermore, the copy number of each identified virus was calculated using the number of virus-associated reads. Specifically, copy number was calculated by multiplying the number of individual virus- or viroid-associated reads by $151 \mathrm{bp}$ and then dividing that value by the size (bp) of the corresponding viral genome. The results of the copy number were in accordance with those of viral abundance (Fig. 5D).

Confirmation of the presence of identified viruses by RT-PCR. RT-PCR with specific primers was performed to confirm the presence of identified viruses (Table 1). The presence of most identified viruses was confirmed using virus-specific primers designed based on a highly conserved region of the $\mathrm{CP}$ gene. However, it was difficult to differentiate between closely related AGCaV and ASPV. Several newly designed primers were tested, and specific primer pairs were selected to detect AGCaV. The RT-PCR products were purified and subjected to Sanger sequencing. BLAST results matched the identified viruses (Supplemen- 


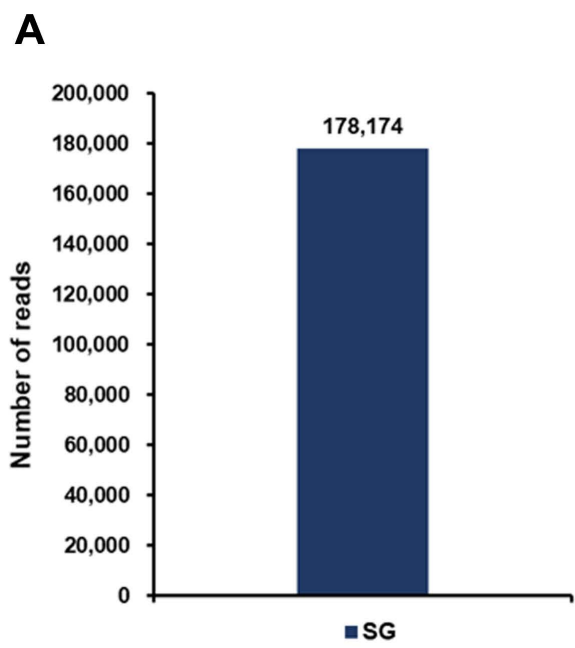

C

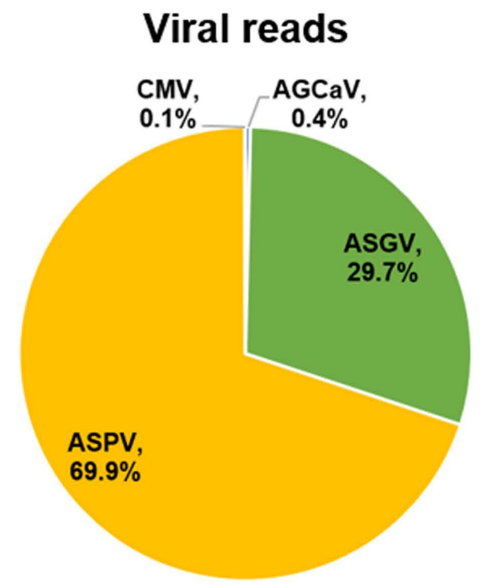

B

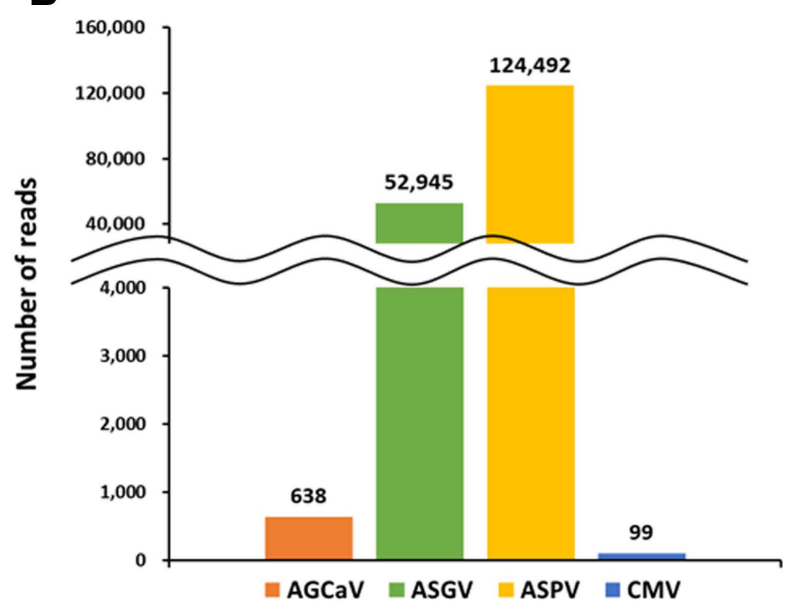

D

\section{Copy number}

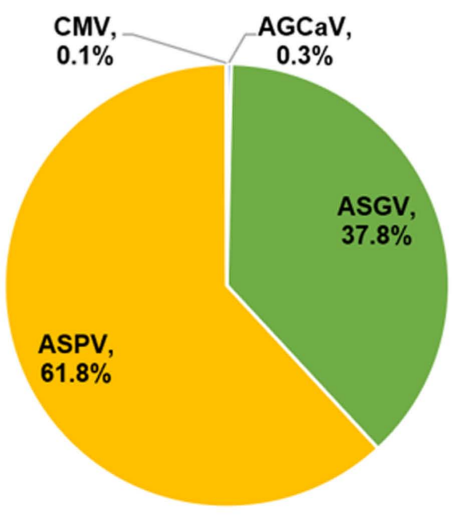

Fig. 5. Abundance and copy number of viruses identified in the pear transcriptome. (A) Number of reads associated with the identified viruses. (B) Number of virus-associated reads in pear transcriptome. (C) Portion of the identified viruses based on the number of virusassociated reads in pear transcriptome. (D) Portion of the identified viruses based on their copy numbers in pear transcriptome. AGCaV, apple green crinkle associated virus; ASGV, apple stem grooving virus; ASPV, apple stem pitting virus; CMV, cucumber mosaic virus.

Table 1. Information of primers used for RT-PCR

\begin{tabular}{|c|c|c|c|c|c|}
\hline Virus & Size (bp) & Position & Primer name & Sequences & Reference \\
\hline \multirow[t]{2}{*}{ ACLSV } & 509 & $6845-6865$ & ACLSV-F31 & GCAGACCCCTTCATGGAAAGA & Bae (2015) \\
\hline & & $7333-7354$ & ACLSV-R31 & CGCAAAGATCAGTCGTAACAGA & \\
\hline \multirow[t]{2}{*}{ AGCaV } & 320 & $1956-1974$ & AGCaV-F & ACCACAGTTGGATGTAATG & In this study \\
\hline & & $2256-2273$ & AGCaV-R & TTCСТCATTATCCTTGTG & \\
\hline \multirow[t]{2}{*}{ ASGV } & 714 & $5641-5664$ & ASGV-F & ATGAGTTTGGAAGACGTGCTTCAA & Shim et al. (2006) \\
\hline & & $6329-6354$ & ASGV-R & CTAACCСТCCAGTTCCAAGTTACT & \\
\hline \multirow[t]{2}{*}{ ASPV } & 367 & $8837-8857$ & ASPV-F001 & AAGCATGTCTGGAACCTCATG & Bae et al. (2015) \\
\hline & & $9178-9202$ & ASPV-R001-2 & GATCAACTTTACTAAAAAGCATAAGT & \\
\hline \multirow[t]{2}{*}{ CMV } & 321 & $1400-1421$ in RNA 3 & CMV-F & TGGTCGTCCAACTATTAACCAC & Choi et al. (1999) \\
\hline & & 1698-1720 in RNA 3 & CMV-R & TACTGATAAACCAGTACCGGTGA & \\
\hline \multirow[t]{2}{*}{ nad5 } & 181 & $1836-1838$ & nad5-F & GATGCTTCTTGGGGCTTCTTGTT & Menzel et al. (2002) \\
\hline & & $1973-1995$ & nad5-R & CTCCAGTCACCAACATTGGCATAA & \\
\hline
\end{tabular}

RT-PCR, real-time polymerase chain reaction. 
tary Fig. 1).

\section{Discussion}

Metatranscriptomic analysis of the virome of a major pear cultivar (SG) in Korea was performed. NGS is a recently emerged tool for virus detection and screening and has been used to discover numerous viruses, viral strains, and viral pathogens responsible for conditions of unknown etiologies (Jo et al., 2018a; Roossinck et al., 2015; Xu et al., 2019). Rapid virus discovery using NGS is considered a modern approach to prevent the outbreaks of novel viral diseases resulting from climate change, introduction via trade, and deterioration of natural ecosystems. Detection of viruses using classic methods, such as ELISA and RTPCR, is often limited in terms of identifying novel virus species (Jeong et al., 2014; Ji et al., 2013). In addition to increasing the rate of virus discovery, NGS has been used to detect viruses and viroids in various fruit trees, including citrus, and peach (Jo et al., 2018b, 2020; Xu et al., 2019).

In the present study, viruses from pooled samples of infected pear leaves were identified and differentiated using a bioinformatics pipeline. Pooling is efficient for identifying viruses that infect plant species across a given geographical distribution. A single plant sample might yield useful information on the virome of a specific plant or population; however, such information is limited in its use for understanding the overall virome of pears in Korea. Pooling also reduces NGS cost. Therefore, pooled libraries and NGS techniques were used to identify viruses in the transcriptomes of a major pear cultivar. Contrary to the previous studies of plant virus metagenomics, the present study investigated RNA viromes of multiple pear plants, thereby facilitating the assessment of viral communities in a specific cultivar.

For virome analyses using NGS, sample preparation is a critical step. Most recent studies have prepared libraries of double-stranded RNAs, mRNAs, virus-derived small interfering RNAs, and plant rRNA-depleted total RNAs from the infected samples (Al Rwahnih et al., 2015; Donaire et al., 2009; Jo et al., 2018b) . Moreover, several recent studies have reviewed the applications of different sample preparation methods and proposed that the selection of nucleic acid extraction methods for HTS depends on diverse viral genome organizations and replication strategies (Wu et al., 2015). Consistent with other studies, massive viral RNA reads obtained from rRNA-depleted total RNA could be de novo assembled into viral genomes. In addition, length of the viral contigs is a crucial factor in the identification of viruses using RNA-Seq. Thus, BLAST results obtained for short contigs can provide misleading information for virus identification. Indeed, in the present study, BLAST analysis indicated the presence of two viruses, namely ASGV and ASPV, whose complete or nearly complete viral genome sequences were obtained.

Moreover, $\mathrm{AGCaV}$ and $\mathrm{CMV}$ were identified from the SG library, in addition to various viruses (e.g., american plum line pattern virus, citrus tatter leaf virus, and prunus necrotic ringspot virus) identified by BLAST analysis of short contigs. However, BLAST results for such short sequences sometimes lead to misidentification, even though partial sequences are derived from a single viral sequence (Jo et al., 2017). Although several studies have identified novel viruses of stone fruits using NGS (Jo et al., 2018a, 2020), no novel viruses or viroids of pear was identified in the present study. Nevertheless, the obtained information is useful for documenting the virosphere diversity of pear trees.

Nonetheless, there are some notable limitations to our employed pooling strategy, despite its cost-effectiveness. For instance, even though the procedure used a consensus viral genome sequence assembled from RNA-Seq data, viral sequence reads may be misaligned and recombination events may be misinterpreted due to pooling of multiple viral isolates (Schlötterer et al., 2015). Thus, pathological assays are required to investigate the concordance between the assembled viruses and sample pathogenicity using indicator woody plants such as pear trees showing susceptibility to pear viruses. In the present study, back inoculation of healthy pear trees based on Koch's postulates couldn't perform, since most pear viruses are transmitted through rooting, cutting, budding, and grafting, not by mechanical inoculation and are spread through infected propagules. Instead, we attempted to test back-inoculation to healthy herbaceous indicator plants such as Chenopodium quinoa, C. amaranticolor, Nicotiana benthamiana, N. tabacum, $N$. rustica, and $N$. glutinosa using mechanical inoculation. However, typical viral symptoms were not observed in the inoculated plants for 2 months (data not shown).

In the present study, phylogenetic analysis of viral genomes generated from the transcriptome data showed that ASGV and ASPV were closely related to isolates from China and Japan. Previous studies of phylogenetic relationships among ASPV isolates, analyzed using CP or triple gene block sequences, reported that the isolates were always clustered according to their hosts (Ma et al., 2016).

RNA viruses that infect plants typically exhibit high mutation rates, and many variants, which together comprise a quasispecies (Schneider and Roossinck, 2001), can often be observed in a single infected plant. In the present study, 
the quasispecies represented by pear tree-infecting viruses were examined by generating assembled virus sequences and analyzing the single-nucleotide variants (SNVs) of the two virus species. Because SNVs of the viruses were detected in library from pooled samples, the SNV information did not represent an individual virus. Even though several studies have reported the mutation rates of various viruses in plants, the present study detected relatively few SNVs (0-92) and relatively low mutation rates $(0-1.42 \%)$, which suggests low levels of genetic diversity in the viromes of pear trees (Jo et al., 2017, 2018a).

In Korea, ASGV is highly prevalent, particularly in the SG cultivar, and it can lead to severe economic losses (Nam and Kim, 1994). Moreover, concomitant infection with multiple viruses is common in plants, and collectively, such viral infections can lead to substantial economic and yield losses (Katwal et al., 2016). To characterize virus populations infecting the SG pear cultivars, viral reads and copy numbers were calculated. Based on the transcriptome data, ASGV and ASPV were found to be the dominant viruses of the tested cultivar, although the copy number of ASPV was almost two-fold higher than that of ASGV.

Finally, RT-PCR was performed to confirm the presence of the identified viruses. ASGV, ASPV, AGCaV, and CMV were detected in SG samples. The present study demonstrated that HTS is a simple tool for identifying potential causative viruses prior to biological tests.

In summary, the present study describes the virome of $\mathrm{SG}$, a major pear cultivar in Korea, and provides a comprehensive overview of the diversity of viral communities in this cultivar. HTS, bioinformatics and phylogenetic analyses, and viral RNA abundance in viromes can provide valuable information on the complexity of genetic variability of disease-causing viruses of pear. The present HTS-based metatranscriptomic analysis furthers genomic information on viruses infecting pear, enabling rapid response to emerging viral diseases of this fruit tree.

\section{Conflicts of Interest}

No potential conflict of interest relevant to this article was reported.

\section{Acknowledgments}

This work was supported by Korea institute of Planning and Evaluation for Technology in Food, Agriculture, Forestry and Fisheries (IPET) through (Agri-Bioindustry Technology Development Program), funded by Ministry of Agriculture, Food and Rural Affairs (MAFRA) (No.
317006-04-2-HD030).

\section{Electronic Supplementary Material}

Supplementary materials are available at The Plant Pathology Journal website (http://www.ppjonline.org/).

\section{References}

Akinyemi, I. A., Wang, F., Zhou, B., Qi, S. and Wu, Q. 2016. Ecogenomic survey of plant viruses infecting Tobacco by next generation sequencing. Virol. J. 13:181.

Al Rwahnih, M., Daubert, S., Golino, D., Islas, C. and Rowhani, A. 2015. Comparison of next-generation sequencing versus biological indexing for the optimal detection of viral pathogens in grapevine. Phytopathology 105:758-763.

Bae, Y. 2015. Development of multiplex RT-PCR for apple viruses and viroid and the incidence of apple viral disease in Gyeongsangbuk-do. M.S. thesis. Kyungpook National University, Daegu, Korea

Bao, Y., Chetvernin, V. and Tatusova, T. 2012. Pairwise sequence comparison (PASC) and its application in the classification of filoviruses. Viruses 4:1318-1327.

Cho, I. S., Kim, D. H., Kim, H. R., Chung, B. N., Cho, J. D. and Choi, G. S. 2010. Occurrence of pome fruit viruses on pear trees (Pyrus pyrifolia) in Korea. Res. Plant Dis. 16:326-330 (in Korean).

Choi, S. K., Choi, J. K., Park, W. M. and Ryu, K. H. 1999. RTPCR detection and identification of three species of cucumoviruses with a genus-specific single pair of primers. J. Virol. Methods 83:67-73.

Donaire, L., Wang, Y., Gonzalez-Ibeas, D., Mayer, K. F., Aranda, M. A. and Llave, C. 2009. Deep-sequencing of plant viral small RNAs reveals effective and widespread targeting of viral genomes. Virology 392:203-214.

Elzebroek, A. T. G. and Wind, K. 2008. Guide to cultivated plants. CAB International, Oxfordshire, UK. 540 pp.

Jeong, J.-J., Ju, H.-J. and Noh, J. 2014. A review of detection of methods for the plant viruses. Res. Plant Dis. 20:173-181.

Ji, Z., Zhao, X., Duan, H., Hu, T., Wang, S., Wang, Y. and Cao, K. 2013. Multiplex RT-PCR detection and distribution of four apple viruses in China. Acta Virol. 57:435-441.

Jo, Y., Bae, J.-Y., Kim, S.-M., Choi, H., Lee, B. C. and Cho, W. K. 2018a. Barley RNA viromes in six different geographical regions in Korea. Sci. Rep. 8:13237.

Jo, Y., Choi, H., Kim, S.-M., Kim, S.-L., Lee, B. C. and Cho, W. K. 2017. The pepper virome: natural co-infection of diverse viruses and their quasispecies. BMC Genomics 18:453.

Jo, Y., Choi, H., Lian, S., Cho, J. K., Chu, H. and Cho, W. K. 2020. Identification of viruses infecting six plum cultivars in Korea by RNA-sequencing. PeerJ 8:e9588.

Jo, Y., Lian, S., Chu, H., Cho, J. K., Yoo, S.-H., Choi, H., Yoon, J.-Y., Choi, S.-K., Lee, B. C. and Cho, W. K. 2018b. Peach RNA viromes in six different peach cultivars. Sci. Rep. 8:1844. 
Katwal, V. S., Handa, A., Thakur, P. D. and Tomar, M. 2016. Prevalence and serological detection of apple viruses in Himachal Pradesh. Plant Pathol. J. (Faisalabad) 15:40-48.

Kearse, M., Moir, R., Wilson, A., Stones-Havas, S., Cheung, M., Sturrock, S., Buxton, S., Cooper, A., Markowitz, S., Duran, C., Thierer, T., Ashton, B., Meintjes, P. and Drummond, A. 2012. Geneious Basic: an integrated and extendable desktop software platform for the organization and analysis of sequence data. Bioinformatics 28:1647-1649.

Kim, N.-Y., Oh, J., Lee, S.-H., Kim, H., Moon, J. S. and Jeong, R.-D. 2018. Rapid and specific detection of apple stem grooving virus by reverse transcription-recombinase polymerase amplification. Plant Pathol. J. 34:575-579.

Lu, Y., Yao, B., Wang, G. and Hong, N. 2018. The detection of ACLSV and ASPV in pear plants by RT-LAMP assays. $J$. Virol. Methods 252:80-85.

Mahfoudhi, N., El Air, M., Moujahed, R., Salleh, W. and Djelouah, K. 2013. Occurrence and distribution of pome fruit viruses in Tunisia. Phytopathol. Mediterr. 52:136-140.

Ma, X., Hong, N., Moffett, P. and Wang, G. 2016. Genetic diversity and evolution of Apple stem pitting virus isolates from pear in China. Can. J. Plant Pathol. 38:218-230.

Menzel, W., Jelkmann, W. and Maiss, E. 2002. Detection of four apple viruses by multiplex RT-PCR assays with coamplification of plant mRNA as internal control. J. Virol. Methods 99:81-92.

Nam, K. W. and Kim, C. H. 1994. Studies on the pear abnormal leaf spot disease. 1. Occurrence and damage. Korean J. Plant Pathol. 10:169-174 (in Korean).

Roossinck, M. J., Martin, D. P. and Roumagnac, P. 2015. Plant virus metagenomics: advances in virus discovery. Phytopathology 105:716-727.

Saito, T. 2016. Advances in Japanese pear breeding in Japan. Breed. Sci. 66:46-59.
Schneider, W. L. and Roossinck, M. J. 2001. Genetic diversity in RNA virus quasispecies is controlled by host-virus interactions. J. Virol. 75:6566-6571.

Schlötterer, C., Kofler, R., Versace, E., Tobler, R. and Franssen, S. U. 2015. Combining experimental evolution with nextgeneration sequencing: a powerful tool to study adaptation from standing genetic variation. Heredity (Edinb) 114:431440.

Shim, H.-K., Hwang, K.-H., Shim, C.-K., Son, S.-W., Kim, D., Choi, Y.-M., Chung, Y., Kim, D.-H., Jee, H.-J. and Lee, S.-C. 2006. The pear black necrotic leaf spot disease virus transmitted by Talaromyces flavus display pathogenicity similar to Apple stem grooving virus strains. Plant Pathol. J. 22:255259.

Tamura, K., Peterson, D., Peterson, N., Stecher, G., Nei, M. and Kumar, S. 2011. MEGA5: molecular evolutionary genetics analysis using maximum likelihood, evolutionary distance, and maximum parsimony methods. Mol. Biol. Evol. 28:27312739.

Wylie, S. J., Li, H., Saqib, M. and Jones, M. G. 2014. The global trade in fresh produce and the vagility of plant viruses: a case study in garlic. PLoS ONE 9:e105044.

Wu, Q., Ding, S.-W., Zhang, Y. and Zhu, S. 2015. Identification of viruses and viroids by next-generation sequencing and homology-dependent and homology-independent algorithms. Annu. Rev. Phytopathol. 53:425-444.

Xu, Y., Li, S., Na, C., Yang, L. and Lu, M. 2019. Analyses of virus/viroid communities in nectarine trees by next-generation sequencing and insight into viral synergisms implication in host disease symptoms. Sci. Rep. 9:12261.

Yoon, J. Y., Joa, J. H., Choi, K. S., Do, K. S., Lim, H. C. and Chung, B. N. 2014. Genetic diversity of a natural population of apple stem pitting virus isolated from apple in Korea. Plant Pathol. J. 30:195-199. 